\title{
Image Compression Using Tap 9/7 Wavelet Transform and Quadtree Coding Scheme
}

\author{
Manal F. Younis* Loay E. George** \\ Received 3, January, 2011 \\ Accepted 20, May, 2011
}

\begin{abstract}
:
This paper is concerned with the design and implementation of an image compression method based on biorthogonal tap-9/7 discrete wavelet transform (DWT) and quadtree coding method. As a first step the color correlation is handled using YUV color representation instead of RGB. Then, the chromatic sub-bands are downsampled, and the data of each color band is transformed using wavelet transform. The produced wavelet sub-bands are quantized using hierarchal scalar quantization method. The detail quantized coefficient is coded using quadtree coding followed by Lempel-Ziv-Welch (LZW) encoding. While the approximation coefficients are coded using delta coding followed by LZW encoding. The test results indicated that the compression results are comparable to those gained by standard compression schemes.
\end{abstract}

Key words: Image Compression, JPEG2000, Wavelet Transform Tap 9/7, Quadtree, LZW

\section{Introduction:}

Compression of digital data is based on various computational algorithms, which can be implemented either in software or in hardware. Compression techniques are classified into two categories: (a) lossless, and (b) lossy approaches. Lossless techniques are capable of recovering the original representation perfectly. Lossy techniques involve algorithms, which recover the presentation similar to the original one. The lossy techniques provide higher compression ratios, and, therefore, they are more often applied to image and video compression than lossless techniques [1].

Currently, the most common form of image compression is known as JPEG 2000 offers numerous advantages over the old JPEG standard, and several of these advantages will be discussed. One main advantage is that JPEG 2000 offers both lossy and lossless compression in the same file stream, while JPEG usually only utilizes lossy compression. JPEG does have a lossless compression engine, but it is separate from the lossy engine, and is not used very often. When high quality is a concern, JPEG 2000 proves to be a much better compression tool. Because of the way the compression engine works, JPEG 2000 promises a higher quality final image, even when using lossy compression. Since the JPEG 2000 format includes much richer content than existing JPEG files, the bottom line effect is the ability to deliver much smaller files that still contain the same level of detail as the larger original JPEG files [2]. Unfortunately, JPEG 2000 is much more complex than older standards and

*University of Baghdad, Computer Dept./ College of Engineering

**University of Baghdad, Computer Dept./College of Science 
low contrast, but it is simpler than other standards [3].

JPEG 2000 is a wavelet-based image compression standard and coding system. The biorthogonal filter (Tap 9/7) was chosen as the basis of the JPEG2000 lossy image compression standard for still images. The coefficients of this filter are given as floating-point numbers. The float filter can be factorized in order to speed up the convolution step. It is primarily suited to high visual quality compression. The use of floating-point arithmetic in the DWT, and the associated rounding errors, make it unsuitable for strictly lossless compression [4].

In this paper we propose an image compression scheme based on Wavelet Transform Tap 9/7 to decompose the image signal into approximate and detail parts, then each parts is coded using set of spatial and statistical encoder which can fit the signal characteristics more appropriately.

\section{Quadtree}

Quadtree technique for data compression is the simplicity of its approach. The quadtree is powerful and simple data structure for representing 2D arrays characterized by high occurance of certain set of symbols (like in case of sparse matrices). It is possible to find applications of quadtree in many different contexts, such as the compression of sub-band coefficients in wavelet decomposition and coding of the sub-blocks data. The quadtree algorithms are based on simple averages and comparisons. A quadtree is a tree-like data structure where each node either terminates on leaf containing useful information, or branches for sub-level quadtrees [5].

The main disadvantage of QuadTree is it takes up a lot of space and the transmitting high levels, only, of the tree gives you a rough image.

\section{LZW}

The LZW data compression algorithm is a powerful technique for lossless data compression that gives high compression efficiency for text as well as image data. The implementation of any compression technique is the most important tasks for any software developer. LZW compression method is simple and is dictionary based. The data set scanned for a sequence of repetitive data occurring. These sequences are stored in the dictionary within the compressed file and references are inserted wherever the repetitive data occurs [6]. Before presenting the proposed system several image compression algorithms have been listed below:

In 2008 Mahmood S.A. was proposed system utilize both discrete cosine transform and wavelet transform to encode the image. First, the system transform the color components of the image from (RGB) to (YUV), the $\mathrm{U}$ and $\mathrm{Y}$ bands are downsampled due to their poor spatial resolution, and then the wavelet transform is applied on each color band separately [4].

In 2007 Antonio C. C., Packed O.H. had presented a complete system to perform low memory wavelet image coding. This approach is "line-based" in that the images are read line by line and only the minimum required number of lines is kept in memory [7].

In 2001 Saffor A., Ramli A., was studied wavelet compression that was applied to compress and decompress a digitized chest $\mathrm{x}$-ray image at various compression ratios [8].

In 2009 BABU1 D.V. and ALAMELU2 N.R. presented an approach for an Enhanced Image Compression Method using Partial EZW Algorithm. In this paper, they 
included integer wavelet transformation and region of interest coding to Partial EZW and hence make it more superior to EZW and SPIHT Algorithm and it is proved with the results [9].

In 2004 Tang X., Pearlman W. A. and James W. Modestino proposed an embedded, block-based, image wavelet transform coding algorithm of low complexity [10].

Yang G. and Guo S. (2006) proposed a new lifting scheme of $7 / 5$ biorthogonal wavelet filter banks (BWFB) which include BT 7/5 filter banks of Brislawn and Treiber for image compression applications [11].

In 2009 Quan D. and Sung Y. applied the Cohen-DaubechiesFeauveau (CDF) 9/7-tap wavelet filter adopted of JPEG2000 in lossy compression is implemented by the lifting scheme or by the convolution scheme while the LeGall 5/3-tap wavelet filter adopted in lossless compression is implemented just by the lifting scheme [12].

The rest of this paper is organized as follows; section 2 contains the proposed scheme. The proposed scheme is tested and the results discussed in section 3. Finally some of derived conclusions are listed in section 4.

\section{Proposed Scheme}

This paper aims to propose an image compression scheme using wavelet transform Tap 9/7. Firstly, the color image data (i.e., Red, Green, Blue components) are loaded; each has size $(\mathrm{H} \mathrm{x} \mathrm{W})$, where $\mathrm{H}$ is the height of the image, and $\mathrm{W}$ is the width of it.

The proposed image compression system consists of two units: the first unit is called "Encoding unit" and the second unit is called "Decoding unit". Each one of these two units consists of many parts, as shown in fig (1).

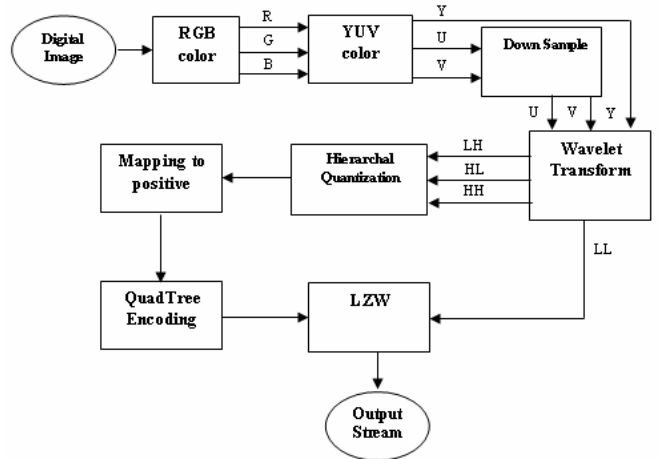

Fig (1): The proposed image compression diagram

The transformation from RGB to YUV is done by the following equations (1) [4]:

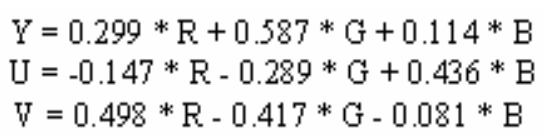

Y component holds more than $80 \%$ of the color image information; $\mathrm{U}$ and $\mathrm{V}$ components hold $10 \%$ in each one. Then the (U, V) components have been down sampled by 2 to get an effective compression [4].

In this proposed method Tap 9/7 wavelet transform was applied on each color transform band $\mathrm{Y}, \mathrm{U}$, and $\mathrm{V}$ to decompose the image data into four sub-bands (LL, LH, HL, and $\mathrm{HH}$ ) each holds certain kind of image information, such that most of the image information is concentrated in the LL sub-band. The following equations illustrate the implemented steps of Tap 9/7 wavelet transform: 


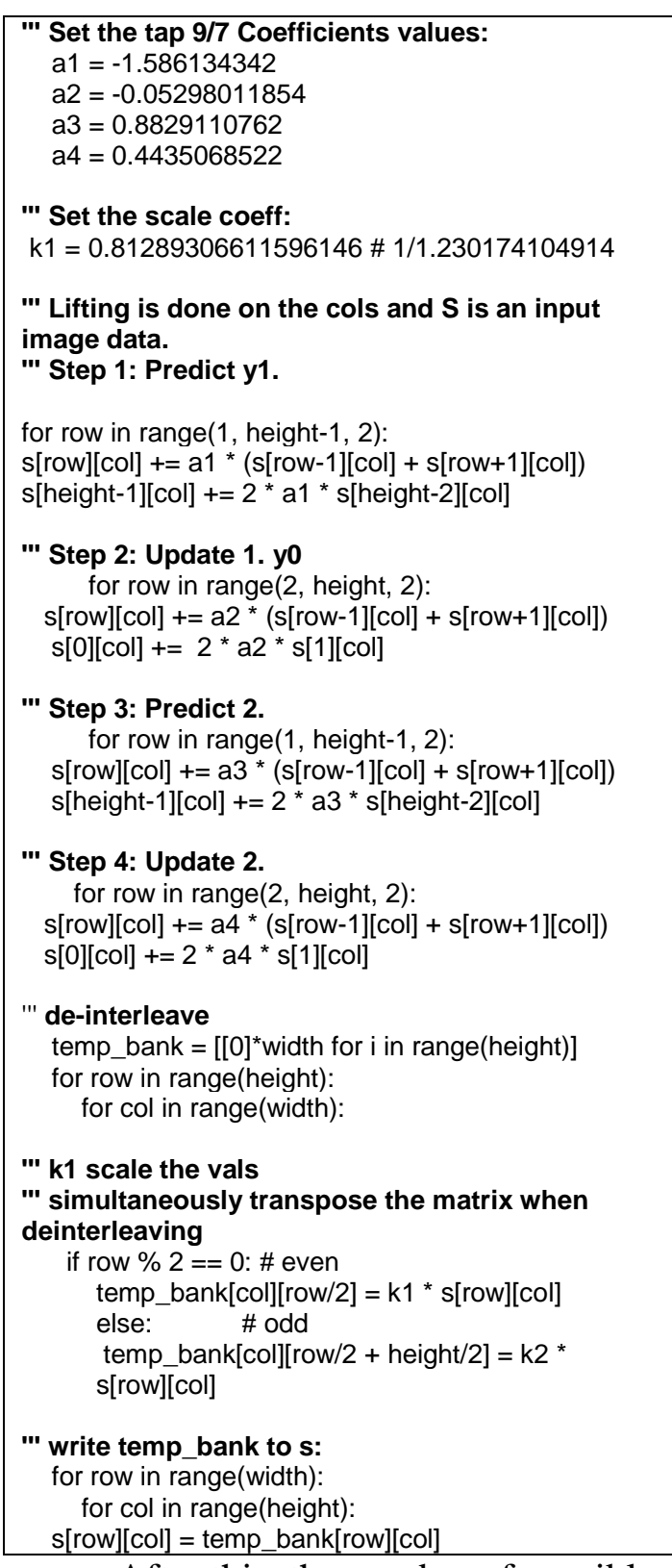

After this, the number of possible values of a wavelet coefficient is reduced by quantization. In our proposed system a hierarchal uniform quantization was used to quantize the coefficients of each wavelet sub-band.

The quantization step used to quantize the coefficients of each sub-band was determined according to the following equation:

$$
\mathrm{Q}_{\text {step }}^{(\mathbf{n})}= \begin{cases}\mathrm{Q} \alpha^{\mathrm{n}-1} & \text { for } \mathrm{LH}, \mathrm{HL} \text { in } \mathrm{n}^{\text {th }} \text { level } \\ \mathrm{Q} \beta \alpha^{\mathrm{n}-1} & \text { for } \mathrm{HH} \text { in } \mathrm{n}^{\text {th }} \text { level }\end{cases}
$$

Where, $\mathrm{n}$ is the wavelet level number (i.e., the pass number), $(\mathrm{Q}, \alpha$, $\beta)$ are quantization parameters (such that, $\mathrm{Q} \geq 1, \alpha \leq 1, \beta \geq 1$ ). According to the above equation the value of the quantization step is reduced with the increase of the wavelet level. The value for HH-subband is taken greater than its corresponding value used to quantize the HL and LH subbands.

The quantization index for each wavelet (detail) coefficient is determined by using the following equation:

$$
\mathrm{T}_{\mathrm{q}}(\mathrm{x}, \mathrm{y})=\operatorname{round}\left(\frac{\mathrm{T}_{\mathrm{w}}(\mathrm{x}, \mathrm{y})}{\mathrm{Q}_{\text {step }}}\right) \ldots 3
$$

Where, $\mathrm{T}_{\mathrm{w}}()$ is the array of the wavelet transform coefficients.

$\mathrm{T}_{\mathrm{q}}($ ) its quantization index array. The below algorithm illustrates the applied quantization process:

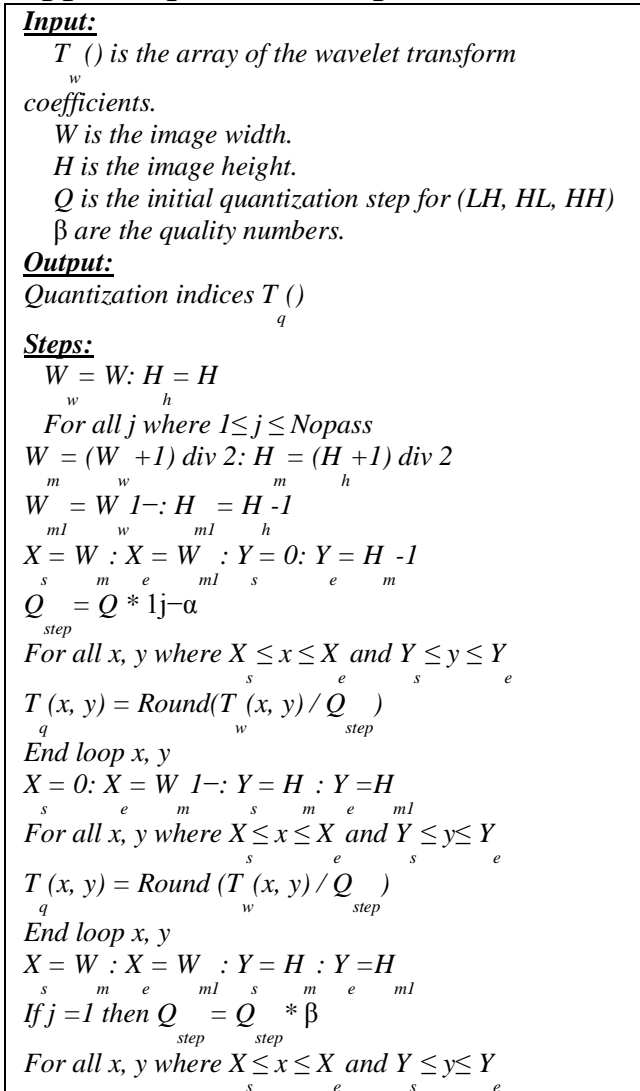




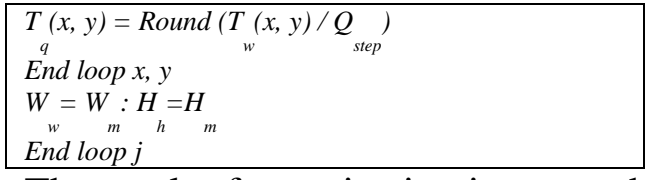

The result of quantization is mapped to positive in order to set the range of quantization coefficient is always positive (i.e., free from negative numbers) to simply to next coding steps.

The below algorithm shows the mapping to positive steps:

\begin{tabular}{|l|}
\hline Input: \\
Tq() is the array of \\
quantization \\
W is the image width \\
H is the image height \\
Output: \\
Positive quantization indices \\
Steps: \\
For all I where $0 \leq I \leq$ \\
BlkSiz- 1 \\
For all $J$ where $0 \leq J \leq$ \\
BlkSiz-1 \\
If Tq $(I, J)>0$ then \\
Tq $(I, J)=2 * T q(I, J)$ \\
Else If Tq $(I, J)<0$ then \\
Tq $(I, J)=2 *$ abs $(T q(I, J))+1$ \\
End if \\
End loop $J$ \\
End loop I
\end{tabular}

Instead of following the JPEG2000 steps, which have high computational complexity. In our prposed system, another set of coding steps was adopted according to low complexity and suitability criteria. The quadtree was adopted to represent the subbands contents.

This paper, introduces the design of an algorithm based on wavelet followed by quadtree code. The proposed quadtree algorithm divides the image into blocks using hierarchal scheme and save them in a way that can restore the blocks again easily.

After this, the data of (LH, HL, and $\mathrm{HH}$ ) sub-bands are compressed using Quadtree algorithm in order to take the advantage of high occurrence of zero values.

The following flowchart shows implemented steps of quadtree coding:

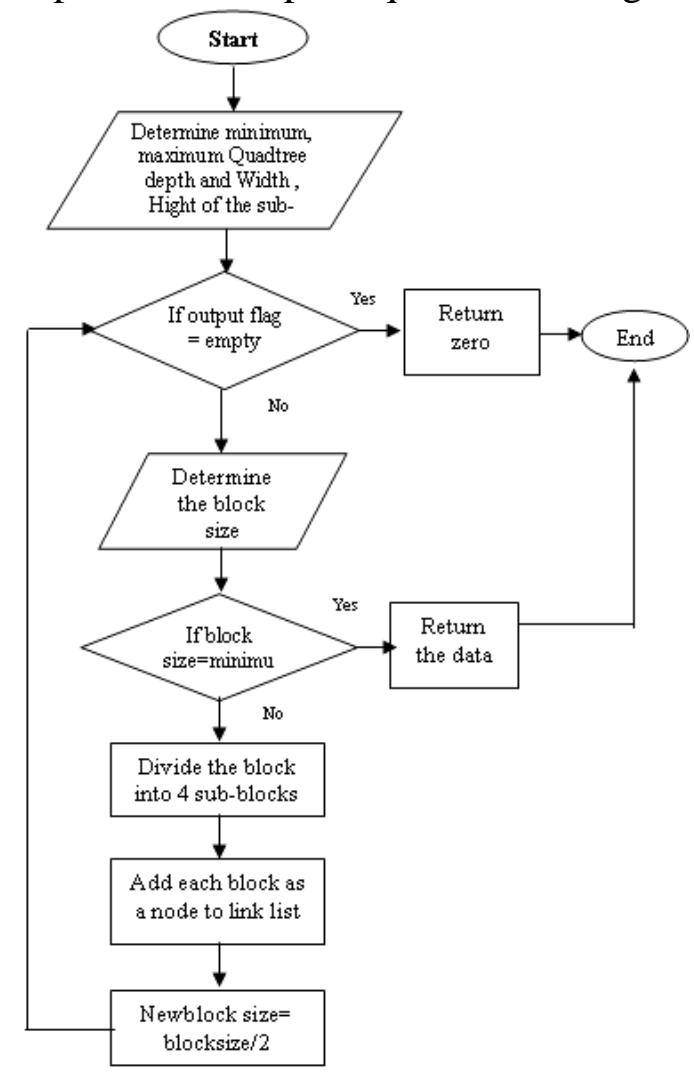

Fig (2): Flowchart of the Quadtree algorithm

Finally, the result of quadtree and the LL sub-band are compressed in LZW coding method. LZW compression process is simple. It replaces strings of characters with single codes. No analysis is done of the incoming text. A new string of characters is added every time it sees to a table of strings. Compression occurs when a single code is output instead of a string of characters. The LZW algorithm output code may be of arbitrary length, but it must have more bits in it than a single character. The first 256 codes are by default assigned to the standard character set. The remaining codes are assigned to strings as the algorithm proceeds. LZW compression is used with different file formats like TIFF and GIF [6]. 
The size of files usually increases to a great extent when it includes lots of repetitive data or monochrome images. LZW compression is the best technique for reducing the size of files containing more repetitive data. LZW compression is fast and simple to apply. Since this is a lossless compression technique, none of the contents in the file are lost during or after compression. The decompression algorithm always follows the compression algorithm. LZW algorithm is efficient because it does not need to pass the string table to the decompression code. The table can be recreated as it was during compression, using the input stream as data. This avoids insertion of large string translation table with the compression data [6].

\section{Testing and Discussion:}

In this paper the Lena image was used as test sample. It is Bitmap image type, of size $\mathbf{5 1 2 \times 5 1 2}$ (pixels). The listed tables show the compression results of the proposed system. In these tables the values of compression ratio (CR), MSE, and PSNR are listed. Their values are affected by the system parameters (i.e., number of wavelet passes, and the quantization parameters values).

Table (1) shows the effects of the quantization parameter QL and $\mathrm{QH}$ on the compression performance parameters, when the value of QL was varied from 1 to 2.5 .

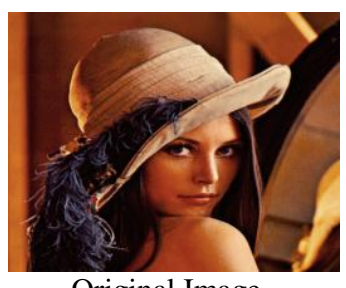

Original Image

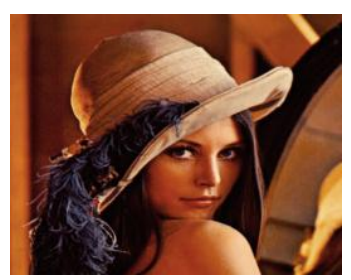

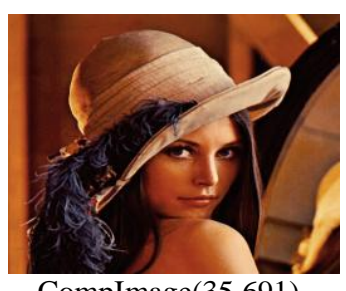

CompImage(35.691)

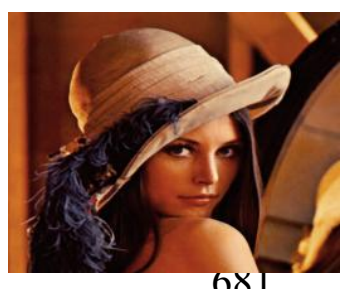

Table (1): The effect of quantization steps $(\mathrm{QL}, \mathrm{QH})$ for the case one wavelet pass $(\alpha=0.75, \beta=1.5)$.

\begin{tabular}{|c|c|c|c|c|}
\hline QL & QH & CR & MSE & PSNR \\
\hline 1 & 2 & 3.080 & 0.494 & 51.192 \\
\hline 1.5 & 2 & 3.099 & 0.807 & 49.064 \\
\hline 2 & 2 & 3.119 & 1.244 & 47.182 \\
\hline 2.5 & 2 & 3.138 & 1.807 & 45.562 \\
\hline 1 & 1 & 2.862 & 0.250 & 54.151 \\
\hline 2 & 5 & 3.837 & 1.975 & 45.174 \\
\hline 2.5 & 10 & 5.170 & 5.689 & 40.581 \\
\hline 2.5 & 15 & 6.425 & 10.568 & 37.891 \\
\hline 2.5 & 20 & 7.471 & 17.530 & 35.693 \\
\hline 2.5 & 30 & 8.753 & 36.225 & 32.541 \\
\hline 2.5 & 40 & 9.257 & 60.773 & 30.294 \\
\hline 2.5 & 60 & 9.459 & 121.354 & 27.290 \\
\hline 2.5 & 80 & 9.462 & 184.470 & 25.472 \\
\hline
\end{tabular}

Table (2) shows the effects of the quantization parameter QL and QH1 on the compression performance parameters, when the value of QL was varied from 1 to 2.5 and QH1 was increased from 1 to 80 .

Table (2): The effect of quantization steps (QL, QH) for the case two wavelet passes $(\alpha=0.75, \beta=1.5)$.

\begin{tabular}{|c|c|c|c|c|}
\hline QL & QH1 & CR & MSE & PSNR \\
\hline 1 & 1 & 2.866 & 0.254 & 54.086 \\
\hline 1.5 & 2 & 3.123 & 0.761 & 49.318 \\
\hline 2 & 5 & 3.984 & 1.838 & 45.486 \\
\hline 2.5 & 10 & 5.709 & 5.083 & 41.070 \\
\hline 2.5 & 15 & 7.634 & 9.274 & 38.458 \\
\hline 2.5 & 20 & 9.504 & 15.226 & 36.305 \\
\hline 2.5 & 30 & 12.234 & 31.300 & 33.175 \\
\hline 2.5 & 40 & 13.473 & 52.470 & 30.932 \\
\hline 2.5 & 60 & 13.969 & 105.057 & 27.917 \\
\hline 2.5 & 80 & 13.943 & 161.204 & 26.057 \\
\hline
\end{tabular}

Figure (3) shows some samples of the decompreesed images, it obvious that the subjective image quality is preserved. 


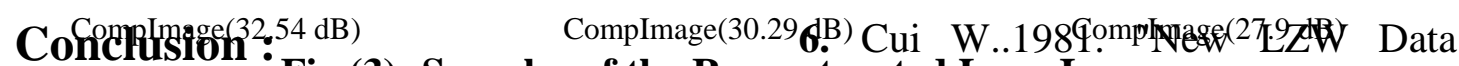

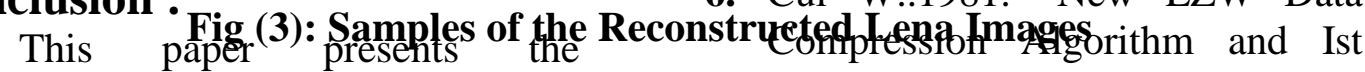
quantization parameters effect of MSE, PSNR and CR. The increase of QL and $\mathrm{QH}$ causes increase in compression ratio (CR). For the case of one wavelet passes the best compressed value was attained when the quantization steps $\mathrm{QL}=2.5$ and $\mathrm{QH}=30$ while for two wavelet passes the best result was obtained when $\mathrm{QL}=2.5$ and $\mathrm{QH} 1=40$.

\section{References:}

1. Furht B., "Image Presentation and Compression", Florida Atlantic University, Department of Computer Science and Engineering, Boca Raton, Florida 33431.

2. Aboufadel E., .2001. "JPEG 2000: The Next Compression Standard using wavelet technology", Grand Valley State University. Proceedings of SPIE, 4115(45):1012.

3. Devaux F. O., Rouvroy G., Legat J.D., Quisquater J.J., and Macq B. . 2006. "A Flexible, Hardware JPEG 2000 Decoder for Digital Cinema Antonin Descampe", Circuits and Systems for Video Technology, IEEE Transactions. 16(1397):1051-8215.

4. Mahmood S.A., .2008. "Using Discrete Cosine Transform to Encode Approximation Wavelet Subband". Nahrin Univercity. Iraq pp 40-45.

5. El-Harby A.A. and G.M. Behery, . 2008. "Qualitative Image Compression Algorithm Relying on Quadtree", Mansoura University, Faculty of Science, Math. Dept.,ICGST-GVIP. 8(3):23.

FPGA Implementation". School info. Sci. Technol. 14(4): 71-76.

7. Antonio C. C., Packard O.H., .2002. "An Algorithm for Low Memory Wavelet Image Compression" Laboratories Integrated Media Systems Center ,University of Southern California Palo Alto, CA 94304-1126 Los Angeles, CA 90089-2564.

8. Saffor A., Ramli A., .2001. "A Comparative Study of Image Compression between JPEG and Wavelet ", Department of Computer and Communication System Faculty of Engineering University Putra Malaysia, 14(1): 39-45.

9. BABU1 D.V., ALAMELU2 N.R., .2009. " Wavelet Based Medical Image Compression Using ROI EZW". International Journal of Recent Trends in Engineering, 1(3):1-3.

10. Tang X., Pearlman W. A. and Modestino J. W., 2004. "Hyperspectral Image Compression Using Three-Dimensional Wavelet Coding: A Lossy-to-Lossless Solution", Center for Image Processing Research.

11. Yang G. and Guo S., .2006. "A New Wavelet Lifting Scheme for Image Compression Applications", Institute of Artificial Intelligence and Robotics of Xi'an Jiaotong University. 10(3):45-56.

12. Quan D. and Sung Y., .2009. "Image Quality Optimization based on Wavelet Filter Design and Wavelet Decompossion in JPEG2000", School of Information \& Mechatronics Gwangju Institute of Science and Technology. 15(4):23-73. 


\section{ضغط الصورة باستخدام التحويل المويجي 9/7 مع طريقة الترميز ذات الشجرة الرباعية}

لؤي ادور جورج**
منال فاضل بيونس*

* جامعة بغداد، كلبة الهندسة / قسم الحاسبات

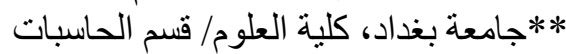

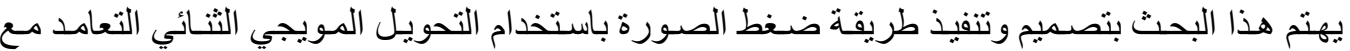

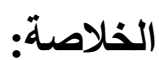

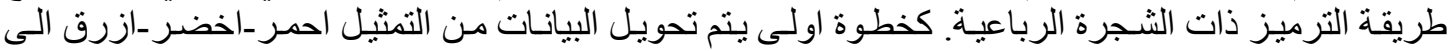

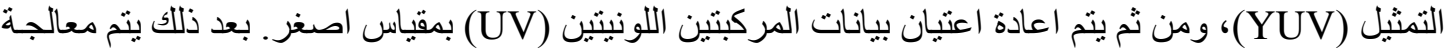

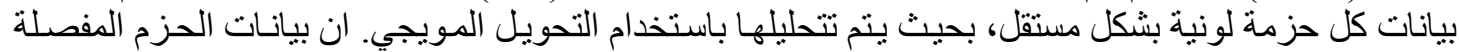

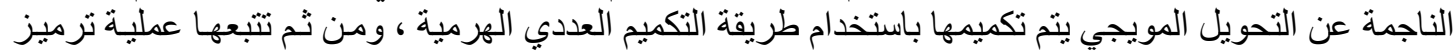

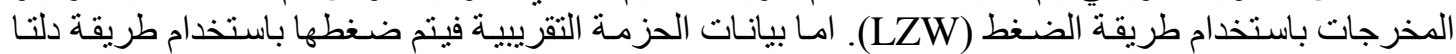

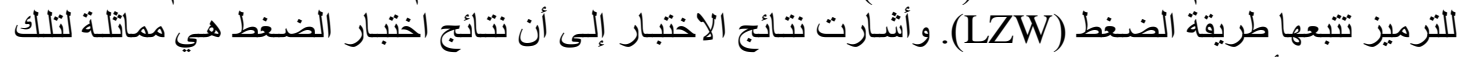
التي اكتسبتها أنظمة الضغط القياسية. 